\title{
NEREIDIDAE (ANNELIDA, POLYCHAETA) COLECTADOS EN LAS CAMPANAAS "FAUNA II, III, IV" (PROYECTO "FAUNA IBÉRICA”) Y CATÁLOGO DE LAS ESPECIES CONOCIDAS PARA EL ÁMBITO IBÉRICO
}

\author{
J. Núñez * y M. C. Brito *
}

\begin{abstract}
RESUMEN
Se confecciona una lista de 19 especies de poliquetos pertenecientes a la familia Nereididae, a partir del material colectado en las campañas oceanográficas "Fauna Ibérica II, III y IV". De éstas, se aportan datos sobre las estaciones de muestreo. De todo el material identificado son nuevas citas para la Península Ibérica tres especies: Ceratonereis vittata Langerhans, 1884, Neanthes rubicunda (Ehlers, 1864) y Nereis perivisceralis Claparède, 1864. También se aporta un catálogo actualizado de los neréididos conocidos para la Península Ibérica compuesto por 36 especies.
\end{abstract}

Palabras clave: Nereididae, Polychaeta, Península Ibérica, catálogo de especies.

\begin{abstract}
Nereididae (Annelida: Polychaeta) collected in the Fauna II, III and IV Cruises ("Fauna Ibérica" Project) and a check-list of known species recorded from the Iberian Peninsula
\end{abstract}

A check-list of 19 polychaetes species belonging to the family Nereididae is made, from the material collected during the Cruises "Fauna Ibérica II, III and IV". Of these, data on the sampling stations are given. As a result of the identification of nereidid specimens, three new records for the Iberian Peninsula were found, Ceratonereis vittata Langerhans, 1884, Neanthes rubicunda (Ehlers, 1864) and Nereis perivisceralis Claparède, 1864. An updated catalogue is also presented, with the 36 nereidid species known for the Iberian Peninsula.

Key words: Nereididae, Polychaeta, Iberian Peninsula, species catalogue.

\section{Introducción}

La familia Nereididae Johnston, 1845, es una de las más representativas y emblemáticas de la macrofauna de poliquetos, utilizada frecuentemente como modelo en el estudio del grupo. Se trata de una familia ampliamente estudiada en el ámbito mundial, de la cual se han descrito 535 especies en 43 géneros (Glasby et al., 2000). En la Península
Ibérica la primera monografía sobre neréididos se debe a Cendrero (1910), que describe 7 especies; más tarde, Campoy (1982) en su Tesis Doctoral sobre las familias de poliquetos "errantes" ibéricas cataloga y describe 22 especies. Actualmente hemos realizado una recopilación bibliográfica de las especies de neréididos citadas para la Península Ibérica y una revisión del material colectado durante las campañas de "Fauna Ibérica II, III y IV" en

* Departamento de Biología Animal (Zoología), Facultad de Biología, Universidad de La Laguna, 38206 La Laguna, Tenerife, Islas Canarias, España 
las cuales se han identificado 19 especies. Como resultado de este trabajo el número de especies se eleva a 36. La familia Nereididae será en el futuro objeto de una publicación monográfica dentro del proyecto "Fauna Ibérica".

En este trabajo se incluye una lista de las estaciones de recolección del material de las tres campañas realizadas dentro del proyecto "Fauna Ibérica". La campaña "Fauna II" se desarrolló en las costas de Galicia, Asturias, Cantabria y País Vasco en el verano de 1991 y la mayoría de las muestras fueron tomadas mediante arrastres bentónicos con Bou de Varas y pelágicos. En "Fauna III" se realizaron los muestreos en las islas Columbretes y Baleares durante el verano de 1994, siendo la mayoría de las muestras obtenidas por buceo con escafandra autónoma, arrastres con Bou de Varas y pelágicos. En "Fauna IV" se continuaron los muestreos en las islas Columbretes y también se hicieron en las islas Hormigas, isla de Alborán y mar circundante, durante el verano de 1996, la mayoría de las muestras se colectaron a buceo con escafandra autónoma, a mano en el intermareal y arrastres bentónicos con Bou de Varas.

El material se encuentra depositado en el Museo Nacional de Ciencias Naturales (MNCN).

\section{Resultados}

La lista de especies se ha ordenado alfabéticamente, cada una de las cuales se acompaña del material examinado, en donde consta la campaña de recolección, referencia de la estación y entre paréntesis el número de ejemplares colectados. Otros datos específicos para cada estación como: nombre de la localidad, coordenadas geográficas, profundidad de captura y tipo de fondo o sustrato quedan reflejadas en el Apéndice final.

Las especies que aparecen marcadas con un $(*)$ se citan por primera vez para la Península Ibérica y, para cada una de ellas, se incluye una breve discusión.

Familia Nereididae Johnston, 1845

Ceratonereis (Composetia) costae (Grube, 1840)

Nereis (ceratonereis) costae, Cendrero (1910): 21, fig. 16-20; Fauvel (1923): 349, fig. 136 a-f.

Material EXAMInAdO.- Fauna III: 178A (1), 190B4 (1), 194A (1), 223B2 (4), 225B (1), 236B1 (1), 236B14 (1), 238A (1), 239A (1), 240B6 (9), 251B (1), 252A (1), 254B (1), 258B3 (2), 259B3 (1), 262B2 (10). Fauna IV: 274B2 (1), 274B4 (1), 274B13 (1), 277B22 (3), 281B14 (1), 282B2 (2), 282B15 (6),
285B2 (1), 285B5 (3), 285B12 (12), 285B22 (1), 290B4 (2), 290B6 (5), 293A (1), 305A (1), 309B (1).

*Ceratonereis (Composetia) vittata Langerhans, 1884

Ceratonereis vittata Langerhans (1884): 254, lám. 15, fig 12; Fauvel (1916): 86, lám. 6, fig. 9-11, lám. 9, fig. 16-20; Núñez (1995): fig. 2 a-g.

Material EXAminado.- Fauna II: 127A (1), 133A (1), 158A (2). Fauna III: 189A (1), 238A (65). Fauna IV: 324A (1).

DisCUSIÓN.- Especie relativamente poco conocida, posiblemente por haberse confundido con otras especies: con Nereis rava (Ehlers, 1864), debido a que presentan un patrón de coloración muy parecido, que persiste después de la fijación de los ejemplares; con Ceratonereis hircinicola (Eisig, 1870), por la morfología de los podios anteriores y similar distribución de los paragnatos, aunque la presencia de una seda homogonfa falciforme en los notopodios de la región media y posterior del cuerpo, la diferencia con claridad de esta última especie. Posiblemente, debido a estas confusiones, las citas de $C$. vittata son tan escasas en el ámbito mundial.

Eunereis longissima (Johnston, 1840)

Eunereis longissima, Fauvel (1923): 351, fig. 138 a-d; HartmannSchröder (1971): 207, fig. 67 a-f; Sardá (1983): 18, fig. 3 a-f; Núñez, Aguirrezabalaga y Ceberio (2000): 34, fig. 6 a-m.

Material eXAminAdo.- Fauna II: 86DL (1), 152A (1).

Micronereis variegata Claparède, 1863

Micronereis variegata, Fauvel (1923): 332, fig. 128 a-f; Núñez, Bacallado y Brito (1981): 163, fig. 2.

Material EXAminAdo.- Fauna III: 218B (1).

Neanthes caudata (delle Chiaje, 1828)

Nereis (Neanthes) caudata, Fauvel (1923): 347, fig. 135 a-e; Núñez, Bacallado y Brito (1981): 170, fig. 7.

Nereis (Neanthiodes) bolivari Rioja (1918): 67, fig.16.

MATERIAL EXAMINADO.- Fauna III: 228A (1), 259B (1), 266A (3).

Neanthes fucata (Savigny, 1820)

Neanthes fucata, Núñez (1995): 77, fig. 5 i.

Nereis fucata, Fauvel (1923): 334, fig. 134 a-f; HartmannSchröder (1971): 205, fig. 66 c-f.

Material eXAMINADO.- Fauna II: 91A(1), 97A(1), 114A (1), 128A (1), 151A (2), 171A (2). Fauna III: 194A (3), 216A (2), 228A (5), 236B3 (1).

Neanthes irrorata (Malmgren, 1867)

Nereis irrorata, Cendrero (1910): 16, figs. 8-15; Fauvel (1914): 170, lám. 13, fig. 9-28; (1923): 340, fig. $132 \mathrm{a}-\mathrm{k}$

Nereis (Neanthes) irrorata, Núñez, Bacallado y Brito (1981): 170, fig. 8.

Material EXAminado.- Fauna III: 251B (1). 
*Neanthes rubicunda (Ehlers, 1864)

Neanthes rubicunda, Núñez (1995): 77, figs. 4 e, 5 g. Lycoris rubicunda, Langerhans (1880): 286, fig. 22

Nereis gisserana Horst (1924): 151, lám. 30, fig. 6, 7; Monro (1939): 394, fig. 302, a-f.

Neanthes aff. gisserana, Núñez, Brito y Bacallado (1984): 18, figs. 3,4 .

Material eXAminado.- Fauna II: 140A (5). Fauna III: 223B7 (1), 230B14 (2), 230B18 (1), 236B3 (1), 236B14 (2), 240B1 (3), 240B4 (5), 248A (2), 252A (1), 262B2 (1), 263B3 (2), 268A (1), 270B1 (1). Fauna IV: 277B11 (1), 281B1 (1), 282B4 (1), 283A (1), 285B1 (3), 285B22 (1), 317A (2).

Discusión.- Ha sido considerada sinónima de $N$. irrorata (Malmgren, 1867) (Fauvel, 1914), aunque las diferencias entre las dos especies son muy evidentes. Las dos especies se diferencian, sobre todo, por la coloración que presentan los ejemplares y la distribución de los paragnatos en las áreas VII-VIII, ya que $N$. rubicunda tiene una sóla fila de paragnatos, mientras que $N$. irrorata tiene dos filas bien marcadas. Ehlers (1864) describe esta especie para las costas italianas, y más tarde Langerhans (1880) la cita por primera vez para Madeira, siendo también conocida de la islas Azores (Fauvel, 1914, en parte como N. irrorata). Núñez (1995) establece la sinonimia entre Neanthes gisserana (Horst, 1924) (Horst, 1924; Monro, 1939) y N. rubicunda, ratificando la validez de este último taxon. Se trata de una especie frecuente en el litoral ibérico, como lo demuestran los numerosos registros obtenidos a partir del material de las campañas de Fauna Ibérica. La confusión en la identificación de esta especie ha sido debida, sin duda, a su errónea sinonimización con N. irrorata.

Nereis falsa Quatrefages, 1865

Nereis falsa, Fauvel (1923): 337, fig. 129 e-m.

Material eXAminado.- Fauna II: 128A (1). Fauna III: 273B1 (1). Fauna IV: 281B3 (1), 285B2 (1), 289P (1).

Nereis funchalensis (Langerhans, 1880)

Nereis funchalensis, Fauvel (1927): 409, fig. 138 h-n.; Acero y San Martín (1986): 15, fig. 9 a, b.

Nereis (Nereis) funchalensis, Núñez, Bacallado y Brito (1981): 167 , fig. 5 .

Nereis (Neanthes) funchalensis, Fauvel (1914): 166, lám. 15, fig. 9-11.

Lycoris funchalensis Langerhans (1880): 287, lám. 15, fig 23 a-n.

Material EXAMINADO.- Fauna III: 273B1 (3).

Nereis lamellosa Ehlers, 1868

Nereis lamellosa, Rioja (1918): 61, fig. 15.

MATERIAL EXAMINADO.- Fauna II: 86DL (8), 152A (1).
Nereis pelagica Linnaeus, 1758

Nereis pelagica, Rioja (1918): 76, fig. 18; Fauvel (1923): 336, fig. $130 \mathrm{a}-\mathrm{f}$.

Material eXAminado.- Fauna III: 262B2 (1).

*Nereis perivisceralis Claparède, 1868

Nereis perivisceralis Claparède (1868): 161, lám. 12, fig 1.

Material eXAminAdo.- Fauna III: 177B2 (1), 179B2 (2), 183B3 (1), 210B5 (1), 211B (1), 213A (1), 218B (1), 229B7 (1), 230B4 (1), 230B18 (1), 230B20 (2), 236B14 (1), 240B1 (1), 240B6 (1), 240B9 (2), 240B10 (4), 250A (1), 258B10 (1), 259B3 (1), 262B2 (1), 267B2 (22), 267B3 (1), 267B4 (1), 270B1 (2), 273B1 (4), 273B2 (1), 273B3 (1), 273B5 (1). Fauna IV: 274B4 (1), 274B10 (3), 281B1 (10), 281B3 (4), 282B2 (2), 282B4 (1), 285B1 (1), 285B22 (1), 299B8 (1), 305A (1).

Discusión.- Se trata de una especie afín a Nereis zonata Malmgren, 1867, siendo la principal diferencia entre las dos especies la distribución de los paragnatos de las áreas VII-VIII: en $N$. perivisceralis hay una sóla fila de paragnatos, mientras que en $N$. zonata existen dos o tres filas. No obstante, $N$. perivisceralis fue sinonimizada con Nereis falsa Quatrefages, 1865 (fide Hartman, 1959), aunque las diferencias entre estas dos especies son muy evidentes, tanto en la distribución de los paragnatos como en la forma de la notoseda homogonfa falcígera. En las islas Azores y costas marroquíes del estrecho de Gibraltar se ha citado la especie Nereis rava $x$ zonata (Bellan, 1978; Amoureux, 1976), cuya descripción nos permite asimilarla a $N$. perivisceralis. En el ámbito íbero-balear ha sido citada como Nereis sp. por Tena (1996) para las islas Chafarinas, siendo ésta la única cita del área. La confusión creada en torno a esta especie se ha debido a su errónea sinonimización, debida a los caracteres intermedios que presenta esta especie entre $N$. rava y $N$. zonata, lo que ha provocado incorrectas identificaciones. En las muestras de las campañas de "Fauna Ibérica" ha resultado ser una especie abundante en dragados.

Nereis rava Ehlers, 1868

Nereis rava, Fauvel (1923): 339, fig. 131 e-f.

MATERIAl EXAMINADO.- Fauna II: 113A (1), 132A (1). Fauna III: 177B1 (1), 179B2 (8), 179B3 (4), 182A (2), 190B7 (1), 194A (1), 197B (1), 200A (1), 213A (1), 217B14 (2), 218B (2), 219A (1), 226B3 (10), 230B14 (2), 230B18 (1), 236B14 (3), 237B1 (1), 238A (2), 240B1 (1), 240B6 (1), 240B8 (2), 240B9 (1), 245A (3), 250A (4), 252A (1), 259B (1), 262B2 (2). Fauna IV: 277B22 (2), 282B2 (1), 298B1 (2), 298B3 (2), 299B8 (1), 305A (12), 323A (1), 324A (3). 
Nereis zonata Malmgren, 1867

Nereis zonata, Fauvel (1923): 338, fig. 130 g-n; Núñez, Bacallado y Brito (1981): 169, fig. 6; Acero y San Martín (1986): 15, fig. 9 c, d.

MATERIAL EXAMINADO.- Fauna III: 179B3 (1), 183 B7 (1), 205B (2), 270B1 (4)

Perinereis cultrifera (Grube, 1840)

Perinereis cultrifera, Fauvel (1923): 352, fig. 137; Campoy (1982): 480, lám. 67.

MATERIAL EXAMINADO.- Fauna III: 204B6 (1), 224B (1), 236B3 (3). Fauna IV: 281B3 (1), 302B5 (1)

\section{Platynereis coccinea (delle Chiaje, 1841)}

Platynereis coccinea, Fauvel (1914): 94, lám. 8, figs. 13, 14; (1923): 360, fig. 141 g-n; Núñez (1995): 80, fig. 3.

MATERIAL EXAMINADO.- Fauna II: 134A (1), 141A (1), 142A (2). Fauna III: 177B1 (2), 179B2 (19), 182A (1), 222A (1), 229B7 (1), 240B10 (1), 250A (2).

Platynereis dumerilii (Audouin y Milne Edwards, 1833) Platynereis dumerilii, Fauvel (1923): 359, fig. 141 a-f.

MATERIAL EXAMINADO.- Fauna II: 119A (1), 140A (6). Fauna III: 177B1 (1), 183B7 (4), 194A (7), 197B (1), 204B6 (1), 218B (4), 224B (38), 230B11 (10), 240B4 (3), 240B9 (1), 251B (1), 259B8 (12), 262B2 (2), 267B2 (1), 267B3 (1), 270B1 (1), 270B4 (1), 271P (9), 272B3 (41). Fauna IV: 274B2 (1), 274B4 (1), 274B10 (8), 275B1 (1), 275B5 (5), 276A (4), 277B1 (2), 277B12 (6), 277B13 (2), 277B15 (2), 277B22 (3), 280P (34), 281B3 (1), 281B4 (6), $281 \mathrm{~B} 14$ (5), 282B2 (1) 283A (2), 285B12 (1), 285B13 (1), 285B22 (1), 287B8 (3), 289P (2), 290B6 (2), 293A (2), 295B20 (1), 299B1 (1), 304A (3), 305A (9), 307A (4), 308B6 (1), 308B7 (2), 309B (2), 313A (1), 315B2 (7), 328B2 (1).

\section{Websterinereis glauca (Claparède, 1870)}

Websterinereis glauca, Pettibone (1971): 27, figs. 14-16; Núñez, Brito y Bacallado (1984): 14, fig. 1.

Leptonereis glauca, Fauvel (1923): 333, fig. 129 a-d.

Material EXAMinado.- Fauna II: 133A (1), 172A (1). Fauna III: 177B1 (4), 194A (2), 213A (1), 218B (3), 230B4 (1). Fauna IV: $323 \mathrm{~A}(1)$

\section{Catálogo de especies citadas para la Península Ibérica}

El presente catálogo registra las especies de neréididos citadas para el ámbito Ibérico y Balear, consta de 36 especies las cuales se han ordenado alfabéticamente. No se incluyen en el presente catálogo las especies: Nereis longisetis McIntosh, 1885 (McIntosh, 1885), ya que la estación de recolección, alejada del estrecho de Gibraltar, la consideramos fuera del ámbito Ibérico-Balear; Nereis jack- soni Kinberg, 1866 (Harmelin, 1964; San Martín, 1982; Sardá, 1984) pues consideramos que las citas de esta especie se realizaron a partir de ejemplares juveniles pertenecientes a $N$. funchalensis (Núñez, 1995); Rullierinereis anoculata Cantone, 1982 fue citada a partir de un sólo ejemplar en mal estado de conservación para las costas peninsulares por Tena et al.(1991), una vez revisado este material la especie ha sido atribuida a Eunereis longissima (Johnston, 1840).

Las principales referencias a partir de las cuales se ha confeccionado este catálogo son las siguientes: Cendrero (1910), Rioja (1917, 1918, 1920), Campoy (1982), Ariño (1987) y Núñez et al., (2000).

Ceratocephale cf. andaman Hylleberg y Nateewathana, 1988

Ceratocephale cf. pacifica (Hartman, 1960)

Ceratocephale loveni Malmgren, 1867

Ceratonereis (Composetia) costae (Grube, 1840)

Ceratonereis (Composetia) hircinicola (Eisig, 1870)

Ceratonereis (Composetia) vittata Langerhans, 1884

Eunereis longissima (Johnston, 1840)

Hediste diversicolor (Müller, 1776)

Micronereis variegata Claparède, 1863

Namanereis littoralis (Grube, 1872)

Neanthes agulhana (Day, 1963)

Neanthes bioculata (Hartmann-Schröder, 1975)

Neanthes caudata (delle Chiaje, 1828)

Neanthes fucata (Savigny, 1820)

Neanthes heteroculata Hartmann-Schröder, 1981

Neanthes irrorata (Malmgren, 1867)

Neanthes kerguelensis (McIntosh, 1885)

Neanthes rubicunda (Ehlers, 1868)

Neanthes succinea (Frey y Leuckart, 1847)

Neanthes virens (Sars, 1835)

Nereis falsa Quatrefages, 1865

Nereis funchalensis (Langerhans, 1880)

Nereis lamellosa Ehlers, 1868

Nereis pelagica Linnaeus, 1758

Nereis perivisceralis Claparède, 1868

Nereis rava Ehlers, 1868

Nereis zonata Malmgren, 1867

Nicon sinica Wu y Sun, 1979

Perinereis cultrifera (Grube, 1840)

Perinereis macropus (Claparède, 1870)

Perinereis marionii (Audouin y Milne Edwards, 1834)

Perinereis oliveirae (Horst, 1889)

Platynereis coccinea (Delle Chiaje, 1841)

Platynereis dumerilii (Audouin y Milne Edwards, 1833)

Platynereis nadiae Abbiati y Castelli, 1992

Websterinereis glauca (Claparède, 1870)

\section{AGRADECIMIENTOS}

El trabajo forma parte de Proyecto "Fauna Ibérica IV", financiado por la CICYT, con el n ${ }^{\circ}$ PB 950235. Nuestro agra- 
decimiento a todas las personas del Museo Nacional de Ciencias Naturales de Madrid que forman parte del mencionado proyecto, por las facilidades que en todo momento nos brindaron para la revisión del material y toma de datos sobre las campañas de recolección de las muestras.

A Guillermo San Martín (Universidad Autónoma de Madrid) y João Gil (Centre d'Estudis Avancats de Blanes) agradecemos sus observaciones y valiosas sugerencias referidas a la primera versión del presente trabajo, que han servido para mejorarlo notablemente.

\section{Referencias}

Acero, M.I. y San Martín, G., 1986. Poliquetos epibiontes del primer horizonte de algas fotófilas en las provincias de Cádiz y Málaga. Estudio faunístico comparado. Boletín de la Real Sociedad Española de Historia Natural (Sección Biológica), 82: 5-24.

Amoureux, L., 1976. Une intéressante collection de Néréidiens (Annélides Polichètes) des côtes marocaines du détroit de Gibraltar. Nereis moroccensis, espèce nouvelle pour la Science. Bulletin du Muséum National d'Histoire Naturelle, sér. 3, 370, zoologie 258: 337-349.

ARIÑO, A., 1987. Bibliografía Ibérica de poliquetos. Base de datos y catálogo de especies. Publicaciones de Biología de la Universidad de Navarra, serie zoológica, 16, EUNSA, Pamplona, 169 pp.

Claparède, É., 1868. Les Annélides Chétopodes du Golfe de Naples. Mémoires de la Société de Physique, Genéve, 19, 20: 1-500 + 31 pl.

Bellan, G., 1978. Une petite collection d'annélides polychètes recoltées dans l'Île de São Miguel (Archipel des Açores). Boletim da Sociedade Portuguesa de Ciências Naturais, 18: 57-67.

CAmpoy, A., 1982. Fauna de España. Fauna de Anélidos Poliquetos de la Península Ibérica. Ediciones de la Universidad de Navarra. Pamplona. 780 pp.

Cendrero, O., 1910. Descripción de algunas especies de Nereidos de las costas Norte y Noroeste de España, principalmente de Santander. La Enseñanza. Madrid. 46 pp.

EHLERS, E., 1864. Die Borstenwümer (Annelida Chaetopoda) nach systematischen und anatomischen Untersuchungen, vol. 1. Wilhelm Engelmann. Leipzig. $748 \mathrm{pp}$.

Fauvel, P., 1914. Annélides Polychètes non-pelagiques provenant des campagnes de l'Hirondelle et de la Princesse Alice (1885-1910). Résultats des Campagnes Scientifiques accomplies par le Prince Albert I de Monaco, 46: 1-432.

FAuvel, P., 1916. Annélides Polychètes pelagiques provenant des campagnes des yachts Hirondelle et Princesse Alice (1885-1910). Résultats des Campagnes Scientifiques accomplies par le Prince Albert I de Monaco, 46: 1-432.
Fauvel, P., 1923. Polychètes Errantes. In: Faune de France, vol. V. Le Chevalier. Paris, 488 pp.

Fauvel, P., 1927. Polychètes Sedentaires. In: Faune de France, vol. VI. Le Chevalier. París, 494 pp.

Glasby, C.J., Hutchings, P.A., Fauchald, K., Paxton, H., Rouse, G.W., Watson Russell, C. \& Wilson, R.S.,2000. Class Polychaeta. In: Beesley, P.L., B. Ross, G.J y Glasby, C.J. (eds.) Polychaetes and Allies: The Southern Synthesis. Fauna of Australia. Vol. 4A. Polychaeta, Myzostomida, Pogonophora, Echiura, Sipuncula. CSIRO Publishing. Melbourne: 1-296.

Harmelin, J.G., 1964. Étude de l'endofaune des "mattes" d'herbiers de Posidonia oceanica Delile. Recuieil des Travaux de la Station Marine d'Endoumme, 35(51): 43-105.

HaRTMAN, O., 1959. Catalogue of the polychaetous annelids of the world. Allan Hancock Foundation Publications, 23:1-627.

HARTMANN-Schröder, G., 1971. Annelida, Borstenwürmer, Polychaeta. In: Die Tierwelt Deutschands, vol. 58. Gustav Fischer. Jena, 594 pp.

Horst, R., 1924. Polychaeta Errantia of the SibogaExpedition. Pt. Nereidae and Hesionidae. SibogaExpedition, Leyden, 99 (2410): 145-198.

LANGERHANS, P., 1880. Die Wurmfauna Madeiras. II. Zeitschrift für Wissenschaftliche Zoologie, 33: 267316.

LANGERHANS, P. 1884. Die Wurmfauna von Madeira. IV. Zeitschrift für Wissenschaftliche Zoologie, 40: $247-$ 285 .

McIntosh, W., 1885. Report on the Annelida Polychaeta collectec by "H.M.S. Challenger" during the years 1873-76. Report on the Scientific Results of the Voyage of H. M. S. Challenger, Zoology, 12: 1-554.

Monro, C. C. A., 1939. On some tropical Polychaeta in the British Museum mostly collected by Dr. C. Crossland at Zanzibar, Thaiti and the Marquesas. III Family Nereidae. Novitates Zoologicae, 41:394-405.

NúÑEZ, J., 1995. Aportaciones sobre cinco especies de Nereidos (Polychaeta, Nereidae) y clave para la identificación de las especies presentes en Canarias. Vieraea, 24: 71-85.

Núñez, J., Aguirrezabalaga, F. y Ceberio, A. 2000. Species of Nereididae from the Capbreton Canyon (Bay of Biscay, Northeast Atlantic). Bulletin of Marine Science, 67(1): 25-37.

NúÑEZ, J., BaCallado, J.J. y BRito, M.C., 1981. Nereidae (Polychaeta Errantia) de las costas del Archipiélago Canario. Boletín del Instituto Español de Oceanografía, 4: 162-177.

NúÑEz, J., BRito, M.C. y BACAllado, J.J. 1984. Nueva contribución al conocimiento de la familia Nereidae (Annelida, Polychaeta), con la descripción de una nueva especie para las Islas Canarias. Anales de la Facultad de Ciencias, 10(1-2): 13-24. 
Pettibone, M. H., 1971. Revision of some species referred to Leptonereis, Nicon and Laeonereis (Polychaeta: Nereididae). Smithsoniam Contributions to Zoology, 104: 1-53.

RIOJA, E., 1917. Datos para el conocimiento de la fauna de Anélidos Poliquetos del Cantábrico. Trabajos del Museo de Ciencias Naturales (Zoología), 29: 1-111.

RiojA, E., 1918. Datos para el conocimiento de la fauna de Anélidos Poliquetos del Cantábrico (2 parte). Trabajos del Museo de Ciencias Naturales (Zoología), 37: 1-99.

RIOJA, E., 1920. Una campaña biológica en el Golfo de Valencia. Anales del Instituto General y Técnico de Valencia, 20: 1-36.

San Martín, G., Acero, M., Contonente, M. y Gómez, J., 1982. Una colección de Anélidos Poliquetos de las costas Mediterráneas Andaluzas. Actas del II Simposio Ibérico del Bentos Marino, 3: 171-182.

SARDÁ, R., 1983. Nuevas citas de Anélidos Poliquetos para la Península Ibérica. Publicaciones del Departamento de Zoología de la Universidad de Barcelona, 9: 13-21
SARDÁ, R., 1984. Estudio sobre la fauna de Anélidos Poliquetos de las zonas Mediolitoral e Infralitoral, en la Región del Estrecho de Gibraltar. Tesis Doctoral. Universidad de Barcelona. Barcelona 901 pp.

TenA, J., 1996. Faunística y Ecología de los Anélidos Poliquetos de los fondos de Sustrato Duro del Archipiélago de las Chafarinas (S. W. Mar de Alborán). Tesis Doctoral (Inédita). Universitat de València. València. 549 pp.

Tena, J., CAPaCcioni-Azzati, R., Porras, R. y TorresGavilÁ, F.J., 1991. Cuatro especies de poliquetos nuevas para las costas mediterráneas españolas en los sedimentos del antepuerto de Valencia. Miscel-lània Zoològica, 15: 29-41. 
Apéndice.— Estaciones de "Fauna Ibérica II, III, IV".

Appendix.- Sampling stations of the Oceanographic Cruises "Fauna Ibérica II, III, IV".

"Fauna II"

\begin{tabular}{|c|c|c|c|c|c|}
\hline Estación & Localidad & Coordenadas iniciales & Coordenadas finales & Profundidad & Sustrato \\
\hline $86 \mathrm{DL}$ & Ría de Muros & $42^{\circ} 41.74^{\prime} \mathrm{N}, 09^{\circ} 10.06^{\prime} \mathrm{E}$ & $42^{\circ} 41.60^{\prime} \mathrm{N}, 09^{\circ} 09.76^{\prime} \mathrm{E}$ & $81-84 \mathrm{~m}$ & Arenoso-fangoso \\
\hline $91 \mathrm{~A}$ & Cabo Finisterre & $42^{\circ} 52.85^{\prime} \mathrm{N}, 09^{\circ} 21.47^{\prime} \mathrm{E}$ & $42^{\circ} 50.93^{\prime} \mathrm{N}, 09^{\circ} 20.91^{\prime} \mathrm{E}$ & $129-133 \mathrm{~m}$ & Pedregoso \\
\hline $97 \mathrm{~A}$ & Ría de Corme y Lage & $43^{\circ} 14.20^{\prime} \mathrm{N}, 09^{\circ} 01.69^{\prime} \mathrm{E}$ & $43^{\circ} 14.13^{\prime} \mathrm{N}, 09^{\circ} 02.14^{\prime} \mathrm{E}$ & $42 \mathrm{~m}$ & Arenoso \\
\hline $113 \mathrm{~A}$ & Gijón & $43^{\circ} 43.57^{\prime} \mathrm{N}, 05^{\circ} 55.67^{\prime} \mathrm{E}$ & $43^{\circ} 43.85^{\prime} \mathrm{N}, 05^{\circ} 56.76^{\prime} \mathrm{E}$ & $116-120 \mathrm{~m}$ & $\begin{array}{l}\text { Fondo coralígeno, } \\
\text { Dendrophyllia ramea }\end{array}$ \\
\hline $114 \mathrm{~A}$ & Gijón & $43^{\circ} 37.71^{\prime} \mathrm{N}, 05^{\circ} 37.59^{\prime} \mathrm{E}$ & $43^{\circ} 38.36^{\prime} \mathrm{N}, 05^{\circ} 39.78^{\prime} \mathrm{E}$ & $72-74 \mathrm{~m}$ & Arenoso-fangoso \\
\hline $119 \mathrm{~A}$ & Cabo Lastres & $43^{\circ} 31.57^{\prime} \mathrm{N}, 05^{\circ} 13.08^{\prime} \mathrm{E}$ & $43^{\circ} 32.26^{\prime} \mathrm{N}, 05^{\circ} 14.14^{\prime} \mathrm{E}$ & $46-48 \mathrm{~m}$ & Arenoso \\
\hline $127 \mathrm{~A}$ & Cabo Mayor & $43^{\circ} 35.83^{\prime} \mathrm{N}, 03^{\circ} 44.32^{\prime} \mathrm{E}$ & $43^{\circ} 35.71^{\prime} \mathrm{N}, 03^{\circ} 44.45^{\prime} \mathrm{E}$ & $130 \mathrm{~m}$ & Rocoso y pedregoso \\
\hline $128 \mathrm{~A}$ & Cabo Mayor & $43^{\circ} 35.92^{\prime} \mathrm{N}, 03^{\circ} 47.53^{\prime} \mathrm{E}$ & $43^{\circ} 35.63^{\prime} \mathrm{N}, 03^{\circ} 49.21^{\prime} \mathrm{E}$ & $141-142 \mathrm{~m}$ & Rocoso y pedregoso \\
\hline $132 \mathrm{~A}$ & Cabo Mayor & $43^{\circ} 29.20^{\prime} \mathrm{N}, 03^{\circ} 45.48^{\prime} \mathrm{E}$ & $43^{\circ} 28.88^{\prime} \mathrm{N}, 03^{\circ} 44.68^{\prime} \mathrm{E}$ & $23-24 \mathrm{~m}$ & Pedregoso \\
\hline $133 \mathrm{~A}$ & Cabo Mayor & $43^{\circ} 30.75^{\prime} \mathrm{N}, 03^{\circ} 47.67^{\prime} \mathrm{E}$ & $43^{\circ} 30.67^{\prime} \mathrm{N}, 03^{\circ} 47.30^{\prime} \mathrm{E}$ & $33-37 \mathrm{~m}$ & Rocoso \\
\hline $140 \mathrm{~A}$ & Punta del Pescador, Santoña & $43^{\circ} 26.62^{\prime} \mathrm{N}, 03^{\circ} 23.83^{\prime} \mathrm{E}$ & $43^{\circ} 26.71^{\prime} \mathrm{N}, 03^{\circ} 23.90^{\prime} \mathrm{E}$ & $21-22 \mathrm{~m}$ & Rocoso y pedregoso \\
\hline $141 \mathrm{~A}$ & Punta del Pescador, Santoña & $43^{\circ} 27.21^{\prime} \mathrm{N}, 03^{\circ} 23.94^{\prime} \mathrm{E}$ & $43^{\circ} 27.90^{\prime} \mathrm{N}, 03^{\circ} 23.99^{\prime} \mathrm{E}$ & $23-32 \mathrm{~m}$ & $\begin{array}{l}\text { Rocoso y pedregoso- } \\
\text { arenoso }\end{array}$ \\
\hline $142 \mathrm{~A}$ & Punta del Pescador, Santoña & $43^{\circ} 28.04^{\prime} \mathrm{N}, 03^{\circ} 24.16^{\prime} \mathrm{E}$ & $43^{\circ} 28.91^{\prime} \mathrm{N}, 03^{\circ} 24.40^{\prime} \mathrm{E}$ & $36-46 \mathrm{~m}$ & $\begin{array}{l}\text { Rocoso y pedregoso- } \\
\text { arenoso }\end{array}$ \\
\hline $151 \mathrm{~A}$ & Cabo Machichaco & $43^{\circ} 25.29^{\prime} \mathrm{N}, 02^{\circ} 31.05^{\prime} \mathrm{E}$ & $43^{\circ} 25.09^{\prime} \mathrm{N}, 02^{\circ} 29.83^{\prime} \mathrm{E}$ & $82-86 \mathrm{~m}$ & Arenoso \\
\hline $152 \mathrm{~A}$ & San Sebastián & $43^{\circ} 22.59^{\prime} \mathrm{N}, 02^{\circ} 03.70^{\prime} \mathrm{E}$ & $43^{\circ} 22.16^{\prime} \mathrm{N}, 02^{\circ} 05.76^{\prime} \mathrm{E}$ & $104-107 \mathrm{~m}$ & Fango negruzco \\
\hline $158 \mathrm{~A}$ & Cabo de Oyambre & $43^{\circ} 29.43^{\prime} \mathrm{N}, 04^{\circ} 20.89^{\prime} \mathrm{E}$ & $43^{\circ} 29.41^{\prime} \mathrm{N}, 04^{\circ} 20.68^{\prime} \mathrm{E}$ & $136-137 \mathrm{~m}$ & Rocoso y pedregoso \\
\hline $171 \mathrm{~A}$ & Islas Sisargas & $43^{\circ} 21.13^{\prime} \mathrm{N}, 08^{\circ} 46.00^{\prime} \mathrm{E}$ & $43^{\circ} 22.40^{\prime} \mathrm{N}, 08^{\circ} 46.05^{\prime} \mathrm{E}$ & $80-96 \mathrm{~m}$ & Arenoso \\
\hline $172 \mathrm{~A}$ & Banco de Galicia & $42^{\circ} 43.65^{\prime} \mathrm{N}, 11^{\circ} 44.99^{\prime} \mathrm{E}$ & $42^{\circ} 44.70^{\prime} \mathrm{N}, 11^{\circ} 43.36^{\prime} \mathrm{E}$ & $761-768 \mathrm{~m}$ & $\begin{array}{l}\text { Fondo de coral, } \\
\text { Madrepora aculata }\end{array}$ \\
\hline
\end{tabular}

"Fauna III"

\begin{tabular}{|c|c|c|c|c|c|}
\hline Estación & Localidad & Coordenadas iniciales & Coordenadas finales & Profundidad & Sustrato \\
\hline 177B1 & Isla Dragonera & $39^{\circ} 35.66^{\prime} \mathrm{N}, 02^{\circ} 19.58^{\prime} \mathrm{E}$ & - & $3 \mathrm{~m}$ & Pared rocosa \\
\hline $178 \mathrm{~A}$ & Isla Dragonera & $39^{\circ} 37.60^{\prime} \mathrm{N}, 02^{\circ} 19.00^{\prime} \mathrm{E}$ & $39^{\circ} 28.99^{\prime} \mathrm{N}, 02^{\circ} 21.22^{\prime} \mathrm{E}$ & $86 \mathrm{~m}$ & Detrítico \\
\hline 179B2 & Punta Jova & $39^{\circ} 38.50^{\prime} \mathrm{N}, 02^{\circ} 25.13^{\prime} \mathrm{E}$ & - & $10 \mathrm{~m}$ & Extraplomo rocoso \\
\hline 179B3 & Punta Jova & $39^{\circ} 38.50^{\prime} \mathrm{N}, 02^{\circ} 25.13^{\prime} \mathrm{E}$ & - & $9 \mathrm{~m}$ & Extraplomo rocoso \\
\hline $182 \mathrm{~A}$ & Punta de la Foradada & $39^{\circ} 44.33^{\prime} \mathrm{N}, 02^{\circ} 31.86^{\prime} \mathrm{E}$ & $39^{\circ} 45.80^{\prime} \mathrm{N}, 02^{\circ} 33.51^{\prime} \mathrm{E}$ & $72-74 \mathrm{~m}$ & Detrítico \\
\hline 183B3 & Punta de la Foradada & $39^{\circ} 45.55^{\prime} \mathrm{N}, 02^{\circ} 37.23^{\prime} \mathrm{E}$ & - & $23 \mathrm{~m}$ & Posidonia oceanica \\
\hline 183B7 & Punta de la Foradada & $39^{\circ} 45.55^{\prime} \mathrm{N}, 02^{\circ} 37.23^{\prime} \mathrm{E}$ & - & $10 \mathrm{~m}$ & Rocoso con Ircinia sp. \\
\hline $194 \mathrm{~A}$ & Cabo de Pera & $39^{\circ} 46.09^{\prime} \mathrm{N}, 03^{\circ} 32.04^{\prime} \mathrm{E}$ & $39^{\circ} 44.17^{\prime} \mathrm{N}, 03^{\circ} 32.45^{\prime} \mathrm{E}$ & $58-59 \mathrm{~m}$ & Detrítico y rocoso \\
\hline 197B & Cabo del Freu & $39^{\circ} 44.93^{\prime} \mathrm{N}, 03^{\circ} 27.62^{\prime} \mathrm{E}$ & - & $8-29 \mathrm{~m}$ & \\
\hline $200 \mathrm{~A}$ & Canal de Menorca & $39^{\circ} 52.30^{\prime} \mathrm{N}, 03^{\circ} 36.65^{\prime} \mathrm{E}$ & $39^{\circ} 55.24^{\prime} \mathrm{N}, 03^{\circ} 37.02^{\prime} \mathrm{E}$ & $85-92 \mathrm{~m}$ & Detrítico y fangoso \\
\hline 204B6 & Cabo Nati & $40^{\circ} 43.25^{\prime} \mathrm{N}, 03^{\circ} 49.50^{\prime} \mathrm{E}$ & - & $13 \mathrm{~m}$ & \\
\hline 205B & Cabo Nati & $40^{\circ} 43.00^{\prime} \mathrm{N}, 03^{\circ} 49.30^{\prime} \mathrm{E}$ & - & - & Supra y mesolitoral \\
\hline 210B5 & Cabo Caballería & $40^{\circ} 05.60^{\prime} \mathrm{N}, 04^{\circ} 40.40^{\prime} \mathrm{E}$ & - & $15 \mathrm{~m}$ & \\
\hline 211B1 & Cala Fornells & $40^{\circ} 02.83^{\prime} \mathrm{N}, 04^{\circ} 08.04^{\prime} \mathrm{E}$ & - & $0-2 \mathrm{~m}$ & \\
\hline $213 \mathrm{~A}$ & Punta de's Murté & $40^{\circ} 04.23^{\prime} \mathrm{N}, 04^{\circ} 11.38^{\prime} \mathrm{E}$ & $40^{\circ} 05.20^{\prime} \mathrm{N}, 04^{\circ} 08.00^{\prime} \mathrm{E}$ & $55-60 \mathrm{~m}$ & Coralígeno \\
\hline $216 \mathrm{~A}$ & Mahón & $39^{\circ} 50.59^{\prime} \mathrm{N}, 04^{\circ} 24.53^{\prime} \mathrm{E}$ & $39^{\circ} 50.10^{\prime} \mathrm{N}, 04^{\circ} 24.60^{\prime} \mathrm{E}$ & $118-119 \mathrm{~m}$ & Rocoso y fangoso \\
\hline 217B14 & Isla del Aire & $39^{\circ} 47.80^{\prime} \mathrm{N}, 04^{\circ} 17.40^{\prime} \mathrm{E}$ & - & $15-20 \mathrm{~m}$ & Rocoso \\
\hline $218 \mathrm{~B}$ & Cabo Font & $39^{\circ} 49.40^{\prime} \mathrm{N}, 04^{\circ} 12.25^{\prime} \mathrm{E}$ & - & $10-23 \mathrm{~m}$ & \\
\hline $219 \mathrm{~A}$ & Cabo Font & $39^{\circ} 47.25^{\prime} \mathrm{N}, 04^{\circ} 14.13^{\prime} \mathrm{E}$ & $39^{\circ} 47.99^{\prime} \mathrm{N}, 04^{\circ} 11.97^{\prime} \mathrm{E}$ & $82-83 \mathrm{~m}$ & Detrítico y fangoso \\
\hline $222 \mathrm{~A}$ & Punta de La Guardia & $39^{\circ} 19.30^{\prime} \mathrm{N}, 03^{\circ} 17.20^{\prime} \mathrm{E}$ & $39^{\circ} 17.82^{\prime} \mathrm{N}, 03^{\circ} 14.16^{\prime} \mathrm{E}$ & $92-97 \mathrm{~m}$ & Detrítico \\
\hline 223B2 & Islote Imperial & $39^{\circ} 07.55^{\prime} \mathrm{N}, 02^{\circ} 57.70^{\prime} \mathrm{E}$ & - & $58 \mathrm{~m}$ & \\
\hline $223 \mathrm{~B} 7$ & Islote Imperial & $39^{\circ} 07.55^{\prime} \mathrm{N}, 02^{\circ} 57.70^{\prime} \mathrm{E}$ & - & $17 \mathrm{~m}$ & \\
\hline $224 B$ & Isla Foradada & $39^{\circ} 12.42^{\prime} \mathrm{N}, 02^{\circ} 58.20^{\prime} \mathrm{E}$ & - & $1-10 \mathrm{~m}$ & \\
\hline $225 \mathrm{~B}$ & Puerto de Cabrera & $39^{\circ} 08.86^{\prime} \mathrm{N}, 02^{\circ} 55.68^{\prime} \mathrm{E}$ & - & $0-5 \mathrm{~m}$ & \\
\hline 226B3 & Cala Galiota & $39^{\circ} 09.28^{\prime} \mathrm{N}, 02^{\circ} 55.30^{\prime} \mathrm{E}$ & - & $8 \mathrm{~m}$ & Rocoso \\
\hline $228 \mathrm{~A}$ & 48 millas de cabo Blanco & $39^{\circ} 15.30^{\prime} \mathrm{N}, 02^{\circ} 23.20^{\prime} \mathrm{E}$ & $39^{\circ} 15.13^{\prime} \mathrm{N}, 02^{\circ} 27.47^{\prime} \mathrm{E}$ & $79-99 \mathrm{~m}$ & Detrítico y rocoso \\
\hline 229B7 & Cabo Blanco & $39^{\circ} 22.10^{\prime} \mathrm{N}, 02^{\circ} 46.80^{\prime} \mathrm{E}$ & - & $10 \mathrm{~m}$ & \\
\hline 230B4 & Islote del Toro & $39^{\circ} 27.74^{\prime} \mathrm{N}, 02^{\circ} 28.42^{\prime} \mathrm{E}$ & - & $14 \mathrm{~m}$ & \\
\hline $230 \mathrm{~B} 11$ & Islote del Toro & $39^{\circ} 27.74^{\prime} \mathrm{N}, 02^{\circ} 28.42^{\prime} \mathrm{E}$ & - & $6 \mathrm{~m}$ & \\
\hline 230B14 & Islote del Toro & $39^{\circ} 27.74^{\prime} \mathrm{N}, 02^{\circ} 28.42^{\prime} \mathrm{E}$ & - & $30 \mathrm{~m}$ & \\
\hline $230 \mathrm{~B} 18$ & Islote del Toro & $39^{\circ} 27.74^{\prime} \mathrm{N}, 02^{\circ} 28.42^{\prime} \mathrm{E}$ & - & $35-43 \mathrm{~m}$ & \\
\hline
\end{tabular}




\begin{tabular}{|c|c|c|c|c|c|}
\hline Estación & Localidad & Coordenadas iniciales & Coordenadas finales & Profundidad & Sustrato \\
\hline 230B20 & Islote del Toro & $39^{\circ} 27.74^{\prime} \mathrm{N}, 02^{\circ} 28.42^{\prime} \mathrm{E}$ & - & $10 \mathrm{~m}$ & \\
\hline 236B1 & Isla de Tagomago & $39^{\circ} 01.80^{\prime} \mathrm{N}, 01^{\circ} 39.14^{\prime} \mathrm{E}$ & - & $36 \mathrm{~m}$ & \\
\hline $236 \mathrm{~B} 3$ & Isla de Tagomago & $39^{\circ} 01.80^{\prime} \mathrm{N}, 01^{\circ} 39.14^{\prime} \mathrm{E}$ & - & $29 \mathrm{~m}$ & \\
\hline $236 \mathrm{~B} 14$ & Isla de Tagomago & $39^{\circ} 01.80^{\prime} \mathrm{N}, 01^{\circ} 39.14^{\prime} \mathrm{E}$ & - & $35-40 \mathrm{~m}$ & \\
\hline $237 \mathrm{~B} 1$ & Losa de Santa Eulalia & $38^{\circ} 58.56^{\prime} \mathrm{N}, 01^{\circ} 35.80^{\prime} \mathrm{E}$ & - & $20 \mathrm{~m}$ & \\
\hline $238 \mathrm{~A}$ & Cabo Martinet & $38^{\circ} 54.07^{\prime} \mathrm{N}, 01^{\circ} 31.14^{\prime} \mathrm{E}$ & $38^{\circ} 56.29^{\prime} \mathrm{N}, 01^{\circ} 32.77^{\prime} \mathrm{E}$ & $55-56 \mathrm{~m}$ & Fangoso \\
\hline $239 \mathrm{~A}$ & Isla Espardel & $38^{\circ} 42.71^{\prime} \mathrm{N}, 01^{\circ} 32.28^{\prime} \mathrm{E}$ & $38^{\circ} 44.90^{\prime} \mathrm{N}, 01^{\circ} 30.51^{\prime} \mathrm{E}$ & $57-58 \mathrm{~m}$ & \\
\hline 240B1 & Punta Galera, Espardel & $38^{\circ} 47.90^{\prime} \mathrm{N}, 01^{\circ} 28.95^{\prime} \mathrm{E}$ & - & $25 \mathrm{~m}$ & \\
\hline 240B4 & Punta Galera, Espardel & $38^{\circ} 47.90^{\prime} \mathrm{N}, 01^{\circ} 28.95^{\prime} \mathrm{E}$ & - & $35 \mathrm{~m}$ & \\
\hline $240 \mathrm{~B} 6$ & Punta Galera, Espardel & $38^{\circ} 47.90^{\prime} \mathrm{N}, 01^{\circ} 28.95^{\prime} \mathrm{E}$ & - & 3-6 m & \\
\hline 240B8 & Punta Galera, Espardel & $38^{\circ} 47.90^{\prime} \mathrm{N}, 01^{\circ} 28.95^{\prime} \mathrm{E}$ & - & $20-25 \mathrm{~m}$ & \\
\hline 240B9 & Punta Galera, Espardel & $38^{\circ} 47.90^{\prime} \mathrm{N}, 01^{\circ} 28.95^{\prime} \mathrm{E}$ & - & $3-5 \mathrm{~m}$ & \\
\hline 240B10 & Punta Galera, Espardel & $38^{\circ} 47.90^{\prime} \mathrm{N}, 01^{\circ} 28.95^{\prime} \mathrm{E}$ & - & $32-35 \mathrm{~m}$ & \\
\hline $245 \mathrm{~A}$ & Banco Emile Baudot & $38^{\circ} 43.03^{\prime} \mathrm{N}, 02^{\circ} 29.51^{\prime} \mathrm{E}$ & $38^{\circ} 43.47^{\circ} \mathrm{N}, 02^{\circ} 30.81^{\prime} \mathrm{E}$ & $140-144 \mathrm{~m}$ & Rocoso \\
\hline $248 \mathrm{~A}$ & 12 millas de Formentera. & $38^{\circ} 45.60^{\prime} \mathrm{N}, 01^{\circ} 51.40^{\prime} \mathrm{E}$ & $38^{\circ} 43.60^{\prime} \mathrm{N}, 01^{\circ} 50.01^{\prime} \mathrm{E}$ & $126-134 \mathrm{~m}$ & Rocoso \\
\hline $250 \mathrm{~A}$ & Cabo Berbería & $38^{\circ} 39.27^{\prime} \mathrm{N}, 01^{\circ} 20.47^{\prime} \mathrm{E}$ & $38^{\circ} 36.70^{\prime} \mathrm{N}, 01^{\circ} 20.03^{\prime} \mathrm{E}$ & $63-66 \mathrm{~m}$ & Pedregoso \\
\hline 251B1 & Cabo Berbería & $38^{\circ} 38.90^{\prime} \mathrm{N}, 01^{\circ} 21.85^{\prime} \mathrm{E}$ & - & $35 \mathrm{~m}$ & \\
\hline $252 \mathrm{~A}$ & Formentera & $38^{\circ} 47.57^{\prime} \mathrm{N}, 01^{\circ} 20.10^{\prime} \mathrm{E}$ & $38^{\circ} 45.17^{\circ} \mathrm{N}, 01^{\circ} 18.97^{\prime} \mathrm{E}$ & $91-92 \mathrm{~m}$ & \\
\hline $254 \mathrm{~B}$ & Estany des Peix & $38^{\circ} 43.46 \mathrm{~N}, 01^{\circ} 24.65^{\prime} \mathrm{E}$ & - & $3 \mathrm{~m}$ & \\
\hline 258B3 & Isla Bleda Mayor & $38^{\circ} 58.32^{\prime} \mathrm{N}, 01^{\circ} 09.90^{\prime} \mathrm{E}$ & - & $6-45 \mathrm{~m}$ & \\
\hline $258 \mathrm{~B} 10$ & Isla Bleda Mayor & $38^{\circ} 58.32^{\prime} \mathrm{N}, 01^{\circ} 09.90^{\prime} \mathrm{E}$ & - & $6-45 \mathrm{~m}$ & \\
\hline $259 \mathrm{~B}$ & Isla Conejera & $38^{\circ} 58.68^{\prime} \mathrm{N}, 01^{\circ} 13.20^{\prime} \mathrm{E}$ & - & $3-38 \mathrm{~m}$ & \\
\hline 259B3 & Isla Conejera & $38^{\circ} 58.68^{\prime} \mathrm{N}, 01^{\circ} 13.20^{\prime} \mathrm{E}$ & - & $35 \mathrm{~m}$ & \\
\hline $259 \mathrm{~B} 8$ & Isla Conejera & $38^{\circ} 58.68^{\prime} \mathrm{N}, 01^{\circ} 13.20^{\prime} \mathrm{E}$ & - & $9 \mathrm{~m}$ & \\
\hline 262B2 & Punta de cala Eubarca & $39^{\circ} 04.52^{\prime} \mathrm{N}, 01^{\circ} 23.13^{\prime} \mathrm{E}$ & - & $10 \mathrm{~m}$ & \\
\hline 263B3 & Punta de cala Eubarca. & $39^{\circ} 04.40^{\prime} \mathrm{N}, 01^{\circ} 21.71^{\prime} \mathrm{E}$ & - & $6-48 \mathrm{~m}$ & $\begin{array}{l}\text { Coralígeno rocoso } \\
\text { y detrítico costero }\end{array}$ \\
\hline $266 \mathrm{~A}$ & 9,8 millas de Columbretes & $39^{\circ} 40.61^{\prime} \mathrm{N}, 00^{\circ} 28.40^{\prime} \mathrm{E}$ & $39^{\circ} 40.44^{\prime} \mathrm{N}, 00^{\circ} 30.85^{\prime} \mathrm{E}$ & $110-113 \mathrm{~m}$ & $\begin{array}{l}\text { Fangoso-arenoso y } \\
\text { pedregoso }\end{array}$ \\
\hline 267B2 & Islas Columbretes & $39^{\circ} 49.84^{\prime} \mathrm{N}, 00^{\circ} 32.32^{\prime} \mathrm{E}$ & - & $32 \mathrm{~m}$ & \\
\hline 267B3 & Islas Columbretes & $39^{\circ} 49.84^{\prime} \mathrm{N}, 00^{\circ} 32.32^{\prime} \mathrm{E}$ & - & $32 \mathrm{~m}$ & \\
\hline 267B4 & Islas Columbretes & $39^{\circ} 49.84^{\prime} \mathrm{N}, 00^{\circ} 32.32^{\prime} \mathrm{E}$ & - & $32 \mathrm{~m}$ & \\
\hline $268 \mathrm{~A}$ & Columbretes & $39^{\circ} 49.65^{\prime} \mathrm{N}, 00^{\circ} 37.57^{\prime} \mathrm{E}$ & $39^{\circ} 52.56^{\prime} \mathrm{N}, 00^{\circ} 37.59^{\prime} \mathrm{E}$ & $82-86 \mathrm{~m}$ & Rocoso y fangoso \\
\hline 270B1 & Isla. Navarrete, Columbretes & $39^{\circ} 53.30^{\prime} \mathrm{N}, 00^{\circ} 39.90^{\prime} \mathrm{E}$ & - & $26-33 \mathrm{~m}$ & \\
\hline 270B4 & Isla. Navarrete, Columbretes & $39^{\circ} 53.30^{\prime} \mathrm{N}, 00^{\circ} 39.90^{\prime} \mathrm{E}$ & - & $3-26 \mathrm{~m}$ & \\
\hline $271 \mathrm{P}$ & Columbrete Grande & $39^{\circ} 53.96^{\prime} \mathrm{N}, 00^{\circ} 40.91^{\prime} \mathrm{E}$ & $39^{\circ} 53.96^{\prime} \mathrm{N}, 00^{\circ} 40.91^{\prime} \mathrm{E}$ & $0 \mathrm{~m}$ & Pelágico \\
\hline 272B3 & Columbrete Grande & $39^{\circ} 53.76^{\prime} \mathrm{N}, 00^{\circ} 41.04^{\prime} \mathrm{E}$ & - & $12 \mathrm{~m}$ & $\begin{array}{l}\text { Rocoso con arena } \\
\text { gruesa }\end{array}$ \\
\hline 273B1 & Columbrete Grande & $39^{\circ} 54.02^{\prime} \mathrm{N}, 00^{\circ} 41.15^{\prime} \mathrm{E}$ & - & $47 \mathrm{~m}$ & $\begin{array}{l}\text { Coralígeno rocoso y } \\
\text { detrítico costero }\end{array}$ \\
\hline 273B2 & Columbrete Grande & $39^{\circ} 54.02^{\prime} \mathrm{N}, 00^{\circ} 41.15^{\prime} \mathrm{E}$ & - & $27-45 \mathrm{~m}$ & $\begin{array}{l}\text { Coralígeno rocoso y } \\
\text { detrítico costero }\end{array}$ \\
\hline 273B3 & Columbrete Grande & $39^{\circ} 54.02^{\prime} \mathrm{N}, 00^{\circ} 41.15^{\prime} \mathrm{E}$ & - & $6-45 \mathrm{~m}$ & $\begin{array}{l}\text { Coralígeno rocoso y } \\
\text { detrítico costero }\end{array}$ \\
\hline 273B5 & Columbrete Grande & $39^{\circ} 54.02^{\prime} \mathrm{N}, 00^{\circ} 41.15^{\prime} \mathrm{E}$ & - & $6-18 \mathrm{~m}$ & $\begin{array}{l}\text { Coralígeno rocoso y } \\
\text { detrítico costero }\end{array}$ \\
\hline
\end{tabular}

"Fauna IV"

\begin{tabular}{|c|c|c|c|c|c|}
\hline Estación & Localidad & Coordenadas iniciales & Coordenadas finales & Profundidad & Sustrato \\
\hline 274B2 & Islas Columbretes & $39^{\circ} 53.56^{\prime} \mathrm{N}, 00^{\circ} 41.04^{\prime} \mathrm{E}$ & $39^{\circ} 53.59^{\prime} \mathrm{N} 00^{\circ} 41.07^{\prime} \mathrm{E}$ & $23 \mathrm{~m}$ & $\begin{array}{l}\text { Rocoso, con Penta- } \\
\text { pora fascialis }\end{array}$ \\
\hline 274B4 & Islas Columbretes & $39^{\circ} 53.56^{\prime} \mathrm{N}, 00^{\circ} 41.04^{\prime} \mathrm{E}$ & $39^{\circ} 53.59^{\prime} \mathrm{N}, 00^{\circ} 41.07^{\prime} \mathrm{E}$ & $22 \mathrm{~m}$ & $\begin{array}{l}\text { Rocoso, con Udotea } \\
\text { y Halimeda }\end{array}$ \\
\hline 274B 10 & Islas Columbretes & $39^{\circ} 53.56^{\prime} \mathrm{N}, 00^{\circ} 41.04^{\prime} \mathrm{E}$ & $39^{\circ} 53.59^{\prime} \mathrm{N}, 00^{\circ} 41.07^{\prime} \mathrm{E}$ & $20 \mathrm{~m}$ & $\begin{array}{l}\text { Rocoso, con Prosthe- } \\
\text { ceraeus sp y Halimeda } \\
\text { tuna }\end{array}$ \\
\hline 274B13 & Islas Columbretes & $9^{\circ} 53.56^{\prime} \mathrm{N}, 00^{\circ} 41.04^{\prime} \mathrm{E}$ & $39^{\circ} 53.59^{\prime} \mathrm{N}, 00^{\circ} 41.07^{\prime} \mathrm{E}$ & $9 \mathrm{~m}$ & Roca suelta \\
\hline 275B1 & Islas Columbretes & $39^{\circ} 53.88^{\prime} \mathrm{N}, 00^{\circ} 41.33^{\prime} \mathrm{E}$ & $39^{\circ} 53.73^{\prime} \mathrm{N}, 00^{\circ} 41.26^{\prime} \mathrm{E}$ & $35 \mathrm{~m}$ & $\begin{array}{l}\text { Detrítico, algas fotó } \\
\text { filas }\end{array}$ \\
\hline 275B5 & Islas Columbretes & $39^{\circ} 53.88^{\prime} \mathrm{N}, 00^{\circ} 41.33^{\prime} \mathrm{E}$ & $39^{\circ} 53.73^{\prime} \mathrm{N}, 00^{\circ} 41.26^{\prime} \mathrm{E}$ & $35 \mathrm{~m}$ & Detrítico \\
\hline $276 \mathrm{~A}$ & Islas Columbretes & $39^{\circ} 53.40^{\prime} \mathrm{N}, 00^{\circ} 39.30^{\prime} \mathrm{E}$ & $39^{\circ} 54.70^{\prime} \mathrm{N}, 00^{\circ} 39.36^{\prime} \mathrm{E}$ & $60-61 \mathrm{~m}$ & Rocas sueltas \\
\hline 277B1 & Islas Columbretes & $39^{\circ} 52.78^{\prime} \mathrm{N}, 00^{\circ} 40.09^{\prime} \mathrm{E}$ & $39^{\circ} 52.72^{\prime} \mathrm{N}, 00^{\circ} 40.06^{\prime} \mathrm{E}$ & $40 \mathrm{~m}$ & Detrítico \\
\hline 277B11 & Islas Columbretes & $39^{\circ} 52.78^{\prime} \mathrm{N}, 00^{\circ} 40.09^{\prime} \mathrm{E}$ & $39^{\circ} 52.72^{\prime} \mathrm{N}, 00^{\circ} 40.06^{\prime} \mathrm{E}$ & $20 \mathrm{~m}$ & Detrítico sobre roca \\
\hline 277B12 & Islas Columbretes & $39^{\circ} 52.78^{\prime} \mathrm{N}, 00^{\circ} 40.09^{\prime} \mathrm{E}$ & $39^{\circ} 52.72^{\prime} \mathrm{N}, 00^{\circ} 40.06^{\prime} \mathrm{E}$ & & Rocoso, algas pardas \\
\hline 277B13 & Islas Columbretes & $39^{\circ} 52.78^{\prime} \mathrm{N}, 00^{\circ} 40.09^{\prime} \mathrm{E}$ & $39^{\circ} 52.72^{\prime} \mathrm{N}, 00^{\circ} 40.06^{\prime} \mathrm{E}$ & $10 \mathrm{~m}$ & Rocoso, Dictyopteris \\
\hline
\end{tabular}




\begin{tabular}{|c|c|c|c|c|c|}
\hline Estación & Localidad & Coordenadas iniciales & Coordenadas finales & Profundidad & Sustrato \\
\hline 277B15 & Islas Columbretes & $39^{\circ} 52.78^{\prime} \mathrm{N}, 00^{\circ} 40.09^{\prime} \mathrm{E}$ & $39^{\circ} 52.72^{\prime} \mathrm{N}, 00^{\circ} 40.06^{\prime} \mathrm{E}$ & & Rocoso, Pinna pernula \\
\hline 277B22 & Islas Columbretes & $39^{\circ} 52.78^{\prime} \mathrm{N}, 00^{\circ} 40.09^{\prime} \mathrm{E}$ & $39^{\circ} 52.72^{\prime} \mathrm{N}, 00^{\circ} 40.06^{\prime} \mathrm{E}$ & $25 \mathrm{~m}$ & Detrítico \\
\hline $280 \mathrm{P}$ & Islas Columbretes & $39^{\circ} 52.38^{\prime} \mathrm{N}, 00^{\circ} 40.07^{\prime} \mathrm{E}$ & - & $0 \mathrm{~m}$ & Pelágico, enjambres \\
\hline 281B1 & Islas Columbretes & $39^{\circ} 52.38^{\circ} \mathrm{N}, 00^{\circ} 40.07^{\prime} \mathrm{E}$ & $39^{\circ} 52.36^{\prime} \mathrm{N}, 00^{\circ} 40.28^{\prime} \mathrm{E}$ & $24 \mathrm{~m}$ & Detrítico \\
\hline 281B3 & Islas Columbretes & $39^{\circ} 52.38^{\prime} \mathrm{N}, 00^{\circ} 40.07^{\prime} \mathrm{E}$ & $39^{\circ} 52.36^{\prime} \mathrm{N}, 00^{\circ} 40.28^{\prime} \mathrm{E}$ & $22 \mathrm{~m}$ & $\begin{array}{l}\text { Conchas de Pinna } \\
\text { nobilis }\end{array}$ \\
\hline 281B4 & I. Columbretes & $39^{\circ} 52.38^{\prime} \mathrm{N}, 00^{\circ} 40.07^{\prime} \mathrm{E}$ & $39^{\circ} 52.36^{\prime} \mathrm{N}, 00^{\circ} 40.28^{\prime} \mathrm{E}$ & $10 \mathrm{~m}$ & $\begin{array}{l}\text { Rocoso, Margaretta } \\
\text { cereoides }\end{array}$ \\
\hline 281B14 & Islas Columbretes & $39^{\circ} 52.38^{\prime} \mathrm{N}, 00^{\circ} 40.07^{\prime} \mathrm{E}$ & $39^{\circ} 52.36^{\prime} \mathrm{N}, 00^{\circ} 40.28^{\prime} \mathrm{E}$ & $19 \mathrm{~m}$ & $\begin{array}{l}\text { Margaretta cereoides } \\
\text { y concreción sobre } \\
\text { Pinna nobilis }\end{array}$ \\
\hline 282B2 & Islas Columbretes & $39^{\circ} 52.68^{\prime} \mathrm{N}, 00^{\circ} 40.23^{\prime} \mathrm{E}$ & $39^{\circ} 52.68^{\prime} \mathrm{N}, 00^{\circ} 40.16^{\prime} \mathrm{E}$ & $23 \mathrm{~m}$ & $\begin{array}{l}\text { Rocoso, concrección } \\
\text { de Pentapora sp }\end{array}$ \\
\hline 282B4 & Islas Columbretes & $39^{\circ} 52.68^{\prime} \mathrm{N}, 00^{\circ} 40.23^{\prime} \mathrm{E}$ & $39^{\circ} 52.68^{\prime} \mathrm{N}, 00^{\circ} 40.16^{\prime} \mathrm{E}$ & $25 \mathrm{~m}$ & $\begin{array}{l}\text { Rocoso, concrección } \\
\text { de Mesophyllum } \\
\text { lichenoides }\end{array}$ \\
\hline 282B15 & Islas Columbretes & $39^{\circ} 52.68^{\prime} \mathrm{N}, 00^{\circ} 40.23^{\prime} \mathrm{E}$ & $39^{\circ} 52.68^{\prime} \mathrm{N}, 00^{\circ} 40.16^{\prime} \mathrm{E}$ & $7 \mathrm{~m}$ & Rocoso \\
\hline $283 \mathrm{~A}$ & Islas Columbretes & $39^{\circ} 53.09^{\prime} \mathrm{N}, 00^{\circ} 36.80^{\prime} \mathrm{E}$ & $39^{\circ} 51.57^{\prime} \mathrm{N}, 00^{\circ} 39.25^{\prime} \mathrm{E}$ & $80 \mathrm{~m}$ & $\begin{array}{l}\text { Fangos terrígenos } \\
\text { costeros y detríticos }\end{array}$ \\
\hline 285B1 & Islas Columbretes & $39^{\circ} 51.27^{\prime} \mathrm{N}, 00^{\circ} 40.55^{\prime} \mathrm{E}$ & $39^{\circ} 51.27^{\prime} \mathrm{N}, 00^{\circ} 40.60^{\prime} \mathrm{E}$ & $42 \mathrm{~m}$ & Detrítico \\
\hline 285B2 & Islas Columbretes & $39^{\circ} 51.27^{\prime} \mathrm{N}, 00^{\circ} 40.55^{\prime} \mathrm{E}$ & $39^{\circ} 51.27^{\prime} \mathrm{N}, 00^{\circ} 40.60^{\prime} \mathrm{E}$ & $42 \mathrm{~m}$ & Detrítico \\
\hline 285B5 & Islas Columbretes & $39^{\circ} 51.27^{\prime} \mathrm{N}, 00^{\circ} 40.55^{\prime} \mathrm{E}$ & $39^{\circ} 51.27^{\prime} \mathrm{N}, 00^{\circ} 40.60^{\prime} \mathrm{E}$ & $9 \mathrm{~m}$ & Rocoso \\
\hline 285B12 & Islas Columbretes & $39^{\circ} 51.27^{\prime} \mathrm{N}, 00^{\circ} 40.55^{\prime} \mathrm{E}$ & $39^{\circ} 51.27^{\prime} \mathrm{N}, 00^{\circ} 40.60^{\prime} \mathrm{E}$ & $15 \mathrm{~m}$ & Esponjas \\
\hline 285B13 & Islas Columbretes & $39^{\circ} 51.27^{\prime} \mathrm{N}, 00^{\circ} 40.55^{\prime} \mathrm{E}$ & $39^{\circ} 51.27^{\prime} \mathrm{N}, 00^{\circ} 40.60^{\prime} \mathrm{E}$ & $12 \mathrm{~m}$ & $\begin{array}{l}\text { Pentapora y } \\
\text { Halimeda sp. }\end{array}$ \\
\hline $285 \mathrm{~B} 22$ & Islas Columbretes & $39^{\circ} 51.27^{\prime} \mathrm{N}, 00^{\circ} 40.55^{\prime} \mathrm{E}$ & $39^{\circ} 51.27^{\prime} \mathrm{N}, 00^{\circ} 40.60^{\prime} \mathrm{E}$ & $30 \mathrm{~m}$ & Meosophyllum \\
\hline 287B8 & Islas Columbretes & $39^{\circ} 51.09^{\prime} \mathrm{N}, 00^{\circ} 40.29^{\prime} \mathrm{E}$ & $39^{\circ} 51.00^{\prime} \mathrm{N}, 00^{\circ} 40.20^{\prime} \mathrm{E}$ & $5 \mathrm{~m}$ & Algas pardas fotófilas \\
\hline 289P & Islas Columbretes & $39^{\circ} 51.40^{\prime} \mathrm{N}, 00^{\circ} 39.63^{\prime} \mathrm{E}$ & - & $0 \mathrm{~m}$ & Pelágico \\
\hline 290B4 & Islas Columbretes & $39^{\circ} 50.86^{\prime} \mathrm{N}, 00^{\circ} 40.60^{\prime} \mathrm{E}$ & $39^{\circ} 50.94^{\prime} \mathrm{N}, 00^{\circ} 40.74^{\prime} \mathrm{E}$ & $12 \mathrm{~m}$ & Rocoso, Maasella sp. \\
\hline 290B6 & Islas Columbretes & $39^{\circ} 50.86^{\prime} \mathrm{N}, 00^{\circ} 40.60^{\prime} \mathrm{E}$ & $39^{\circ} 50.94^{\prime} \mathrm{N}, 00^{\circ} 40.74^{\prime} \mathrm{E}$ & $25 \mathrm{~m}$ & $\begin{array}{l}\text { Rocoso, algas rojas } \\
\text { con incrustaciones }\end{array}$ \\
\hline $293 \mathrm{~A}$ & Islas Columbretes & $39^{\circ} 51.20^{\prime} \mathrm{N}, 00^{\circ} 41.40^{\prime} \mathrm{E}$ & $39^{\circ} 53.50^{\prime} \mathrm{N}, 00^{\circ} 42.00^{\prime} \mathrm{E}$ & $78-81 \mathrm{~m}$ & \\
\hline 295B20 & Islas Columbretes & $39^{\circ} 54.02^{\prime} \mathrm{N}, 00^{\circ} 41.08^{\prime} \mathrm{E}$ & $39^{\circ} 53.98^{\prime} \mathrm{N}, 00^{\circ} 41.11^{\prime} \mathrm{E}$ & $16 \mathrm{~m}$ & \\
\hline 298B1 & Islas Hormigas & $37^{\circ} 39.89^{\prime} \mathrm{N}, 00^{\circ} 38.12^{\prime} \mathrm{W}$ & $37^{\circ} 39.84^{\prime} \mathrm{N}, 00^{\circ} 38.08^{\prime} \mathrm{W}$ & $47 \mathrm{~m}$ & \\
\hline 298B3 & Islas Hormigas & $37^{\circ} 39.89^{\prime} \mathrm{N}, 00^{\circ} 38.12^{\prime} \mathrm{W}$ & $37^{\circ} 39.84^{\prime} \mathrm{N}, 00^{\circ} 38.08^{\prime} \mathrm{W}$ & $41 \mathrm{~m}$ & \\
\hline 299B1 & Islas Hormigas & $37^{\circ} 39.52^{\prime} \mathrm{N}, 00^{\circ} 38.92^{\prime} \mathrm{W}$ & $37^{\circ} 39.47^{\prime} \mathrm{N}, 00^{\circ} 38.86^{\prime} \mathrm{W}$ & $14 \mathrm{~m}$ & Rocoso \\
\hline 299B1 & Islas Hormigas & $37^{\circ} 39.52^{\prime} \mathrm{N}, 00^{\circ} 38.92^{\prime} \mathrm{W}$ & $37^{\circ} 39.47^{\prime} \mathrm{N}, 00^{\circ} 38.86^{\prime} \mathrm{W}$ & $14 \mathrm{~m}$ & Rocoso \\
\hline $304 \mathrm{~A}$ & Isla de Alborán & $35^{\circ} 55.99^{\prime} \mathrm{N}, 03^{\circ} 01.57^{\prime} \mathrm{W}$ & $35^{\circ} 55.94^{\prime} \mathrm{N}, 03^{\circ} 01.68^{\prime} \mathrm{W}$ & $29-35 \mathrm{~m}$ & $\begin{array}{l}\text { Rodolitos, rocas y } \\
\text { detritos gruesos }\end{array}$ \\
\hline $305 \mathrm{~A}$ & Isla de Alborán & $35^{\circ} 55.68^{\prime} \mathrm{N}, 03^{\circ} 03.25^{\prime} \mathrm{W}$ & $35^{\circ} 55.63^{\prime} \mathrm{N}, 03^{\circ} 02.67^{\prime} \mathrm{W}$ & $33-49 \mathrm{~m}$ & Rodolitos calcáreos \\
\hline 307A & Isla de Alborán & $35^{\circ} 55.59^{\prime} \mathrm{N}, 03^{\circ} 02.34^{\prime} \mathrm{W}$ & $35^{\circ} 55.77^{\prime} \mathrm{N}, 03^{\circ} 01.54^{\prime} \mathrm{W}$ & $37-39 \mathrm{~m}$ & Rocoso y rodolitos \\
\hline 308B6 & Isla de Alborán & $35^{\circ} 56.49^{\prime} \mathrm{N}, 03^{\circ} 01.20^{\prime} \mathrm{W}$ & $35^{\circ} 56.49^{\prime} \mathrm{N}, 03^{\circ} 01.20^{\prime} \mathrm{W}$ & $32-34 \mathrm{~m}$ & \\
\hline 308B7 & Isla de Alborán & $35^{\circ} 56.49^{\prime} \mathrm{N}, 03^{\circ} 01.20^{\prime} \mathrm{W}$ & $35^{\circ} 56.49^{\prime} \mathrm{N}, 03^{\circ} 01.20^{\prime} \mathrm{W}$ & $32-34 \mathrm{~m}$ & \\
\hline 309B & Isla de Alborán al & $35^{\circ} 56.52^{\prime} \mathrm{N}, 03^{\circ} 01.72^{\prime} \mathrm{W}$ & $35^{\circ} 56.41^{\prime} \mathrm{N}, 03^{\circ} 01.72^{\prime} \mathrm{W}$ & $10-12 \mathrm{~m}$ & Rocoso \\
\hline $313 \mathrm{~A}$ & Isla de Alborán & $35^{\circ} 49.91^{\prime} \mathrm{N}, 03^{\circ} 14.63^{\prime} \mathrm{W}$ & $35^{\circ} 50.36^{\prime} \mathrm{N}, 03^{\circ} 13.72^{\prime} \mathrm{W}$ & $118 \mathrm{~m}$ & $\begin{array}{l}\text { Fangos arenosos de } \\
\text { plataforma }\end{array}$ \\
\hline 315B2 & Isla de Alborán & $35^{\circ} 58.00^{\prime} \mathrm{N}, 02^{\circ} 58.46^{\prime} \mathrm{W}$ & $35^{\circ} 58.00^{\prime} \mathrm{N}, 02^{\circ} 58.46^{\prime} \mathrm{W}$ & $36 \mathrm{~m}$ & Rocoso \\
\hline $317 \mathrm{~A}$ & Isla de Alborán & $35^{\circ} 52.49^{\prime} \mathrm{N}, 03^{\circ} 08.90^{\prime} \mathrm{W}$ & $35^{\circ} 52.31^{\prime} \mathrm{N}, 03^{\circ} 07.92^{\prime} \mathrm{W}$ & $87-213 \mathrm{~m}$ & $\begin{array}{l}\text { Escarpe rocoso con } \\
\text { piedras }\end{array}$ \\
\hline $323 \mathrm{~A}$ & Isla de Alborán & $35^{\circ} 57.82^{\prime} \mathrm{N}, 03^{\circ} 00.04^{\prime} \mathrm{W}$ & $35^{\circ} 57.50^{\prime} \mathrm{N}, 03^{\circ} 01.16^{\prime} \mathrm{W}$ & $67-79 \mathrm{~m}$ & Rodolitos \\
\hline $324 \mathrm{~A}$ & Isla de Alborán & $35^{\circ} 57.17^{\prime} \mathrm{N}, 03^{\circ} 02.00^{\prime} \mathrm{W}$ & $35^{\circ} 57.18^{\prime} \mathrm{N}, 03^{\circ} 03.00^{\prime} \mathrm{W}$ & $51-58 \mathrm{~m}$ & $\begin{array}{l}\text { Rodolitos y agregados } \\
\text { de Neopycnodonte } \\
\text { cochlear }\end{array}$ \\
\hline 328B2 & Isla de Alborán & $35^{\circ} 57.80^{\prime} \mathrm{N}, 02^{\circ} 58.61^{\prime} \mathrm{W}$ & - & $40 \mathrm{~m}$ & Rocoso \\
\hline
\end{tabular}

